\title{
Identification of hub genes and small-molecule compounds in medulloblastoma by integrated bioinformatic analyses
}

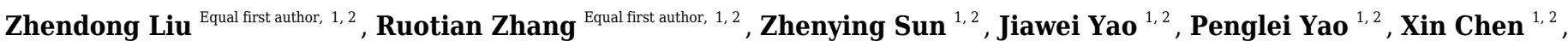 \\ Xinzhuang Wang ${ }^{1,2}$, Ming Gao ${ }^{1,2}$, Jinzhao Wan ${ }^{1,2}$, Yiming Du ${ }^{1,2}$, Shiguang Zhao ${ }^{\text {Corresp. } 1,2}$ \\ ${ }^{1}$ Department of Neurosurgery, The First Affiliated Hospital of Harbin Medical University, Harbin, Heilongjiang Province, People's Republic of China \\ 2 Institute of Brain Science, Harbin Medical University, Harbin, Heilongjiang Province, People's Republic of China \\ Corresponding Author: Shiguang Zhao \\ Email address: guangsz@hotmail.com
}

Background. Medulloblastoma (MB) is the most common intracranial malignant tumor in children. The genes and pathways involved in the pathogenesis of MB are relatively unknown. We aimed to identify potential biomarkers and small-molecule drugs for MB.

Methods. Gene expression profile data sets were obtained from the Gene Expression Omnibus (GEO) database and the differentially expressed genes (DEGs) were identified using the Limma package in R. Functional annotation, and cell signaling pathway analysis of DEGs was carried out using DAVID and Kobas. A protein-protein interaction network was generated using STRING. Potential small-molecule drugs were identified using CMap.

Result. We identified 104 DEGs (29 upregulated; 75 downregulated). Gene ontology analysis showed enrichment in the mitotic cell cycle, cell cycle, spindle, and DNA binding. Cell signaling pathway analysis identified cell cycle, HIF-1 signaling pathway, and phospholipase D signaling pathway as key pathways. SYN1, CNTN2, FAIM2, MT3, and SH3GL2 were the prominent hub genes and their expression level were verified by RT-qPCR. Vorinostat, resveratrol, trichostatin A, pyrvinium, and prochlorperazine were identified as potential drugs for MB. The five hub genes may be targets for diagnosis and treatment of $M B$, and the small-molecule compounds are promising drugs for effective treatment of MB.

Conclusion. In this study we obtained five hub genes of MB, SYN1, CNTN2, FAIM2, MT3, and SH3GL2 were confirmed as hub genes. Meanwhile, Vorinostat, resveratrol, trichostatin A, pyrvinium, and prochlorperazine were identified as potential drugs for MB. 
1 Identification of hub genes and small-molecule compounds in medulloblastoma by

2 integrated bioinformatic analyses

3 Zhendong Liu ${ }^{1,2 *}$, Ruotian Zhang ${ }^{1,2 *}$, Zhenying Sun ${ }^{1,2}$, Jiawei Yao ${ }^{1,2}$, Penglei Yao ${ }^{1,2}$, Xin

4 Chen $^{1,2}$, Xinzhuang Wang ${ }^{1,2}$, Ming Gao ${ }^{1,2}$, Jinzhao Wan ${ }^{1,2}$, Yiming Du ${ }^{1,2}$, Shiguang

$5 \mathrm{Zhao}^{1,2}$

6

$7{ }^{1}$ Department of Neurosurgery, The First Affiliated Hospital of Harbin Medical University, No. 23 Youzhe

8 ng Street, Nangang District, 150001 Harbin, Heilongjiang Province, People's Republic of China

9 Institute of Brain Science, Harbin Medical University, No. 23 Youzheng Street, Nangang District, 15000

101 Harbin, Heilongjiang Province, People's Republic of China

11

12

13

14

15

16

17

18

19

20

21

22

23

24

25

26

27

28

Corresponding Author:

Shiguang Zhao

Department of Neurosurgery, The First Affiliated Hospital

of Harbin Medical University, No. 23 Youzheng Street, Nangang District,150001

Harbin, Heilongjiang Province, People's Republic of China.

Email address: guangsz@,hotmail.com

*These authors contributed equally: Zhendong Liu and Ruotian Zhang

\section{Abstract}

Background. Medulloblastoma (MB) is the most common intracranial malignant tumor in children. The genes and pathways involved in the pathogenesis of MB are relatively unknown. We aimed to identify potential biomarkers and small-molecule drugs for MB. Methods. Gene expression profile data sets were obtained from the Gene Expression Omnibus (GEO) database and the differentially expressed genes (DEGs) were identified using the Limma package in R. Functional annotation, and cell signaling pathway analysis of DEGs was carried out using DAVID and Kobas. A protein-protein interaction 
29 network was generated using STRING. Potential small-molecule drugs were identified 30 using CMap.

31 Result. We identified 104 DEGs (29 upregulated; 75 downregulated). Gene ontology 32 analysis showed enrichment in the mitotic cell cycle, cell cycle, spindle, and DNA 33 binding. Cell signaling pathway analysis identified cell cycle, HIF-1 signaling pathway, 34 and phospholipase D signaling pathway as key pathways. SYN1, CNTN2, FAIM2, MT3, 35 and SH3GL2 were the prominent hub genes and 36 their expression levels were verified by RT-qPCR. Vorinostat, resveratrol, trichostatin A, 37 pyrvinium, and prochlorperazine were identified as potential drugs for MB. The five hub 38 genes may be targets for diagnosis and treatment of $\mathrm{MB}$, and the small-molecule 39 compounds are promising drugs for effective treatment of MB.

40 Conclusion. In this study we obtained five hub genes of MB, SYN1, CNTN2, FAIM2,

41 MT3, and SH3GL2 were confirmed as hub genes. Meanwhile, Vorinostat, resveratrol, 42 trichostatin A, pyrvinium, and prochlorperazine were identified as potential drugs for 43 MB.

\section{Introduction}

45 Medulloblastoma (MB) is one of the most common intracranial malignant tumors in 46 children. Standardized treatment options including maximal surgical resection, radiation 47 therapy, and chemotherapy can improve overall five-year survival rates; however, the 48 prognosis of patients with advanced disease is still unsatisfactory (Kim et al. 2010) (1). 49 Despite extensive studies on the pathogenesis and progression of $\mathrm{MB}$, the 50 pathophysiology of disease development is still unclear. The pathogenesis of MB is

51 closely related to many factors such as mutations in genes, abnormalities in cellular 
52 immunity, and changes in environmental factors (Wang et al. 2018) (2). A deep 53 understanding of the changes in protein expression involved in the pathogenesis of MB is

54 critical for the development of better treatment strategies.

55 Gene chip is a genetic testing technology that has been in use for more than a 56 decade. The gene chip technology can rapidly detect changes in gene expression in the 57 sample and is suitable for screening differentially expressed genes (DEGs) (Vogelstein et 58 al. 2013) (3). In recent years, gene chip technology has been widely used, and data from 59 numerous microarray studies have been stored in free public databases. These databases 60 provide valuable data for further research. So far, several gene chip data have been 61 analyzed, and hundreds of different genes involved in the development of several central 62 nervous system cancers such as gliomas have been identified (Xi et al. 2017; Zeng et al. 63 2018) $(4,5)$. However, most gene chip data is a mixture of both MB and glioma and does 64 not accurately represent changes in $\mathrm{MB}$, and therefore does not generate satisfactory 65 results.

66 There have been reports of microarray data to find DEGs in medulloblastoma.(Shaabanpo 67 ur Aghamaleki $\quad$ et al.

68 2019) However, the reliability of the analysis results was controversial due to the heterog 69 eneity of the sample from a single cohort study. Therefore, most single-chip analyses are 70 not sufficient to identify effective biomarkers in MB. To overcome this, we used data 71 derived from four gene chips to identify effective biomarkers for MB. This method is 72 more accurate and does not have the disadvantages associated with the single-chip 73 analysis. 
74 In this study, we downloaded four MB microarray datasets - GSE42656, 75 GSE74195, GSE109401, and GSE50161 from free public Gene Expression

76 Omnibus database (GEO). A total of $77 \mathrm{MB}$ samples and 39 normal brain samples were 77 included in this study. We identified the DEGs using the Limma package in R software 78 (version 3.5.0) from the four gene expression profiles and subsequently, used the Venny 79 online tool for further integrated analyses. We then employed the DAVID databases and 80 Kobas online tool to identify the functions of the identified DEGs and the key cell 81 signaling pathways involved. The network of protein-protein interaction (PPI) was 82 generated using the STRING database. Finally, we used the CMap database to explore 83 potential small-molecule compounds that can be used for treating MB.

\section{Materials \& Methods}

85 Microarray data information

86 We downloaded the gene expression profile data for GSE42656, GSE74195, 87 GSE109401, and GSE50161 from the GEO database (http://www.ncbi.nlm.nih.gov/geo). 88 The GSE42656 data set was based on the GPL6947 Platforms (Illumina HumanHT-12 89 V3.0 expression beadchip) and contained nine MB samples and 16 normal brain samples 90 (Henriquez et al. 2013)(6). The GSE74195 data set was based on the GPL570 Platform 91 (Affymetrix Human Genome U133 Plus 2.0 Array) and contained 27 MB and five normal 92 brain tissue samples (de Groot et al. 2011) (7). The GSE50161 data set was based on the 93 GPL570 Platform (Affymetrix Human Genome U133 Plus 2.0 Array) and included 22 94 MB samples and 13 normal brain tissues (Griesinger et al. 2013) (8). The GSE109401 95 data set was based on GPL16686 Platforms Affymetrix Human Gene 2.0 ST Array 96 [transcript (gene) version]) and included 19 medulloblastoma samples and five normal 97 brain samples (Rivero-Hinojosa et al. 2018) (9). We selected these four gene expression 
98 profiles for further integrated analyses to avoid racial differences and errors in individual

99 experiments.

100 Identification of DEGs in Medulloblastoma

101 The DEGs were identified based on the series matrix file using Limma package in $\mathrm{R}$ 102 software (version 3.5.0) according to the cut-off standard ( $\mathrm{p}<0.05$ and $\log \mathrm{FC}>1$ ) (Fang 103 et al. 2017) (10). The four sets of differential expression data were respectively divided 104 into upregulated DEmRNAs and downregulated DEmRNAs and were uploaded to Venny 105 (http://bioinfogp.cnb.csic.es/tools/venny/) for integrated analyses. A heat map was 106 generated using the pheatmap package in R. The GSE74195 dataset was used as the 107 reference to generate the heat map. The heatmap showed 29 upregulated DEGs, and 75 108 downregulated DEGs.

GO and KEGG enrichment analysis of DEGs

110 The DAVID online database and Kobas online tool were used for functional and 111 pathway enrichment analyses (Huang da et al. 2009) (11). We uploaded the DEGs to 112 DAVID for GO functional annotation analysis and Kobas for KEGG pathway enrichment 113 analysis. $\mathrm{P}<0.05$ was used as the cut-off value.

114 Building a PPI network

115 The STRING public database, which provides PPI network analysis, can evaluate 116 direct and indirect links between DEGs (http://string-db.org) (Franceschini et al. 2013) 117 (12). The DEGs were uploaded to STRING to obtain the PPI network using an 118 Interaction score of 0.2. Cytoscape was used for visualizing the PPI network and for 119 identifying the hub genes based on the degree of connectivity between DEGs. 
121 CMap, an online tool, can be based on the gene expression profile of a disease to

122 mine potential therapeutic drugs (Lamb et al. 2006) (13). We divided the DEGs of MB

123 into upregulated DEGs and downregulated DEGs, and uploaded them to CMap to explore

124 the drugs. $\mathrm{P}<0.001$ and Enrichment $<-0.8$ was used as the cut-off criteria. The 3D 125 structures of the small-molecule compounds are available from PubChem

126 (https://pubchem.ncbi.nlm.nih.gov/).

127

128

129

130

131

132

133

134

135

136

137

138

139

140

141

143

142 GGGGTGATGTTGCCCTGTAA-3' (sense) and 5'-AGGTCTGAGGCATTGGTTCG-

RNA isolation and reverse transcription

quantitative polymerase chain reaction ( $R T-q P C R)$ analysis

Total

RNA is extracted from in normal human brain tissue and medulloblastoma cell lines (Dao $y$ and D283) using Tri-

Reagent (Sigma, USA) according to the manufacturer 's instructions. NanoDrop One spe ctrophotometer (Thermo Fisher Scientific, USA) was used to evaluate the quality and qua ntity of the RNA. The Transcriptor First Stand cDNA Synthesis Kit (Roche, USA) was us ed to reverse transcribe total RNA into cDNA.The FastStart Universal SYBR Green Mast er (ROX) (Roche, Germany) and the QuantStudio software (Thermo Fisher Scientific, U SA) were reserved for RTqPCR based on the manufacturer 's instructions. The GAPDH gene was used as an endog enous reference.

The primer sequences were as follows: 5'-GGACACGTGCTCAGAGATT-

3' (sense) and 5'-TCTACGATGAGCTGTTTGTCTTC-3'(antisense) for SYN1, 5'3'(antisense) for CNTN2, 5'-CTGATTCTCCTGACCGTCTTTAC-3' (sense) and 5'- 
144 GAACTTGGTCTGGAAGCTGAA-3'(antisense) for FAIM2,5'-

145 CAAGTGCGAGGGATGCAAAT-3' (sense) and 5'-TGGCACACTTCTCACACTCC-

$1463^{\prime}$ (antisense) for MT3, 5'-CTCAGCCTAGAAGGGAATATCAAC-3' (sense) and 5'-

147 CAGCAGGGCTGATCCATTT-3'(antisense) for SH3GL2, and 5'-

148 CACCCACTCCTCCACCTTTGA-3' (sense) and 5'-ACCACCCTGTTGCTGTAGCCA-

$1493^{\prime}$ (anti- sense) for GAPDH. The results were analyzed using the -

$150 \Delta \mathrm{C}$ T method with an unpaired t-test, and a P-

151 value $<0.05$ was considered a meaningful result.

152 Results

153 Screening of DEGs in MB

154 We downloaded the gene expression datasets - GSE42656, GSE109401, 155 GSE50161, and GSE74195 from GEO. From the GSE42656 dataset, we 156 identified 869 DEGs, of which 222 were upregulated, and 647 were downregulated.

157 A total of 764 DEGs were identified from the GSE109401 data set -274 upregulated 158 and 490 downregulated; 5494 DEGs from GSE50161 - 2798 upregulated and 2696 159 downregulated, and 1000 DEGs were identified from GSE74195 - 422 160 upregulated and 578 downregulated. Integrated analyses revealed that 104 DEGs were 161 consistently expressed in the four data sets (Figure. 1A and 1B); these included 29 162 upregulated DEGs, and 75 downregulated DEGs in MB tissue compared to the normal 163 brain tissue (Table 1). A heat map of the DEG distribution was generated using the 164 GSE74195 dataset as a reference(Figure S1).

165 Gene Ontology Analysis of DEGs

166 The identified DEGs were analyzed using DAVID using $\mathrm{p}<0.05$ as the cut-off 167 standard to identify the functions associated with the DEGs. The GO function annotation 
168 is divided into three functional groups - cell component (CC), molecular function

169 (MF), and biological process (BP). As shown in Figure. 2 and Table 2, within the BPs the 170 upregulated DEGs were closely related to mitotic cell cycle, cell cycle phase-M phase,

171 cell cycle process, cell cycle, spindle organization, and microtubule-based process and

172 the downregulated DEGs were closely related to vesicle-mediated transport. Within CC

173 the upregulated DEGs were closely related to the spindle-microtubule, and cytoskeleton,

174 whereas the downregulated DEGs were closely related to the synapse, synapse part, 175 clathrin-coated vesicle, synaptic vesicle, cytoplasmic vesicle, coated vesicle, cytoplasmic 176 membrane-bounded vesicle, vesicle, and membrane-bounded vesicle. Within MF, the 177 upregulated DEGs showed a close relationship with DNA binding and the downregulated 178 DEGs did not show any association.

\section{KEGG pathway analysis}

180 The 104 DEGs were grouped as upregulated and downregulated DEGs and the 181 cellular signaling pathways represented were analyzed by KEGG ( Figure. 3 and Table 182 3). The upregulated genes are mainly involved in the cell cycle, ubiquitin-mediated 183 proteolysis, viral carcinogenesis, one-carbon pool by folate, and microRNAs in cancer. 184 The downregulated genes are mainly involved in the synaptic vesicle cycle, bacterial 185 invasion of epithelial cells, insulin secretion, HIF-1 signaling pathway, phospholipase D 186 signaling pathway, and cell adhesion molecules (CAMs).

\section{PPI network generation and identification of the hub gene}

189 The interrelationships between the 104 DEGs were analyzed using STRING. We found 190 that 100 of the 104 DEGs were related to each other and were visualized using Cytoscape 
$191-100$ nodes and 776 edges are included in the network of PPI and the hub gene

192 based on the degree of connectivity between genes were identified (Figure.4A).

193 Among the hub genes, the most significant hub genes were SYN1, CNTN2, FAIM2, MT3, 194 and SH3GL2 (Figure.4B).

195 Potential therapeutic drugs for MB

196 Of the 104 DEGs, only 85 were eventually converted to IDs from 22,214 probes in 197 the Affymetrix platform. After that, we used CMap and identified six potential 198 therapeutic drugs based on set criteria (Table 4). A PubMed literature search revealed that 199 two drugs - vorinostat and resveratrol - have been reported to have a therapeutic 200 effect in MB. The other three drugs, trichostatin A, pyrvinium, and prochlorperazine, 201 have been reported to have therapeutic effects in other cancers. The 3D structures of 202 these potential therapeutic drugs are available from PubChem (Figure.5).

203 Experiments verify the five hub DEmRNAs in MB

204 To verify the expression levels of the five hub genes in the PPI network, RT-

205 qPCR was used to detect their expression levels in normal human brain tissue and medull 206 oblastoma cell lines (Daoy and D283). The five hub genes in the PPI network, including

207 SYN1,CNTN2, FAIM2, MT3, and SH3GL2, were all down-

208 regulated DEGs in MB, and this result was confirmed by RT-qPCR

209 (Figure 6).This shows that our analysis results were completely credible.

\section{Discussion}

211 Although several studies have been carried out to understand the pathogenesis of MB, 212 there is no effective strategy to reduce the incidence or mortality of MB. This may be 213 because most studies till date have focused on a single genetic event contributing to MB 
214 pathogenesis. Therefore, in order to identify effective molecular markers, we used data 215 derived from four cohorts profile datasets obtained from the GEO database (GSE42656, 216 GSE74195, GSE109401, GSE50161). Integrated analyses of the four data sets revelated 217104 DEGs including 29 upregulated, and 75 downregulated DEGs. Enrichment of these 218 genes identified certain cellular signaling pathways which may provide novel insights to 219 the understanding of the pathogenesis of MB. We also used CMap, a drug development 220 tool, and identified six potential drugs that can be used for the treatment of MB. To our 221 knowledge, this is the first study to use integrated bioinformatical analysis for studying 222 the pathogenesis of MB.

223 GO enrichment analysis showed that the upregulated DEGs were mainly involved in 224 the spindle, cell cycle phase, $M$ phase, cell cycle, spindle organization, microtubule 225 cytoskeleton and the downregulated DEGs were mainly closely related to clathrin-coated 226 vesicle, synaptic vesicle, and cytoplasmic vesicle. These results are consistent with 227 previous literature reports that spindle defects play a vital role in the pathological process 228 of MB (Abdelfattah et al. 2018) (14). LCL161 an inhibitor of apoptosis proteins (IAP) 229 combined with chemotherapy can slow MB cell proliferation by inducing G2/M phase 230 arrest (Chen et al. 2018) (15). Patupilone, a microtubule stabilizer, can reduce clonogenic 231 survival and enhance the therapeutic efficacy of radiotherapy effect in MB (Oehler et al. 232 2011) (16). a-synuclein binds to cytoplasmic vesicles to change the surface 233 morphology of in U251 glioblastoma cells (Duan et al. 2017) (17). Synaptic vesicle 234 protein $2 \mathrm{~A}$ is a predictor of the efficacy of levetiracetam in glioma patients (de Groot et al. 235 2011)(18). The internalization of clathrin-coated vesicles can reduce the sensitivity of 236 metabotropic receptors in C6 cells (Luis Albasanz et al. 2002) (19). 
237 KEGG pathway analysis demonstrated that the upregulated genes have significant 238 enrichment in pathways including one-carbon pool by folate, microRNAs in cancer, 239 DNA replication, and basal transcription factors. Previous studies have shown that these 240 signaling pathways are mainly involved in the development of cancer. For example, the 241 basal transcription factors such as oncogene Orthodenticle Homeobox 2, play an 242 important role in cell migration and proliferation in MB (Wortham et al. 2014) (20). 243 Folate receptor (Folr1) participates in the pathway network of the one-carbon pool by 244 folate, which is related to clinical, pathological, neuroimaging features, and prognosis of 245 MB patients (Liu et al. 2017) (21). MicroRNA-31 inhibits DNA replication by targeting 246 minichromosome maintenance complex component 2 (MCM2), which has a strong 247 inhibitory effect on MB growth (Jin et al. 2014) (22). The downregulated genes were 248 mainly involved in HIF-1 signaling pathway, phospholipase D signaling pathway, and 249 phosphatidylinositol signaling system. These signaling pathways are involved in the 250 pathological process of cancer. For example, phosphatidylinositol signaling system, such 251 as the phosphatidylinositol 3-kinase (PI3K) pathway is associated with reduced survival 252 in patients and is one of the most common signaling pathway-related abnormality in 253 gliomas (Tuncel \& Kalkan 2018) (23). Phospholipase D signaling pathway specifically 254 inhibits autophagic flux and decreases GBM cell viability (Bruntz et al. 2014) (24). The 255 hypoxia-inducible factor-1 signaling pathway is vital for the invasion and metastasis of 256 glioma cells (Yaghi et al. 2016) (25). Together, our results show that the cellular 257 pathways of DEGs identified in this study are closely related to the development of 258 cancer. 
260 of the hub genes selected in this network have been reported to be closely related to the 261 pathological process of cancers. For example, SYN1, one of the neuronal genes, is related 262 to the formation and maintenance of synaptic contacts and the expression of REST. 263 RCOR1 may cause deregulated expression of SYN1, which contributes to the 264 maintenance of glioblastoma stem-like cells (GSC) (Yucebas et al. 2016) (26). CNTN2, a 265 cell adhesion protein, is a downstream protein of RACK1, which affects the growth and 266 differentiation of glioma cells through the RTK/Ras/MAPK signaling pathway (Yan \& 267 Jiang 2016) (27). FAIM2 is an anti-apoptotic molecule that promotes tumor cell growth 268 through Fas-mediated mechanisms. Knock-down of FAIM2 can significantly affect 269 tumor cell proliferation in small-cell lung cancer (Kang et al. 2016) (28). MT3 is a tumor 270 suppressor gene, and its expression is significantly reduced in AML samples. The 271 overexpression of MT3 can inhibit cell proliferation and promote tumor cell apoptosis in 272 pediatric acute myeloid leukemia (Tao et al. 2014) (29). SH3GL2, as a suppressor for 273 tumors, and has reduced expression in glioma tissues promoting migration and infiltration 274 of glioma cells by enhancing STAT3/MMP2 signaling (Zhu et al. 2017) (30). These five 275 hub genes have important regulatory effects in the pathophysiology of cancers, but their 276 role has not been reported in MB. These hub genes are potential targets for the treatment 277 of MB.

278 CMap is an online tool that can be used to identify potential therapeutic drugs based on 279 DEGs in various disease (Lamb et al. 2006) (13). In the current study, we identified six 280 drugs using CMap analysis. Two of these drugs have been previously reported to have a 281 therapeutic effect on MB. The combination of vorinostat and MLN8237 can significantly 
282 inhibit the proliferation of MB cells (Muscal et al. 2013) (31). Resveratrol inhibits the 283 growth of cancer cells by regulating the Notch signaling pathway to promote apoptosis 284 and differentiation of MB cells (Wang et al. 2008) (32). Three drugs - trichostatin A, 285 pyrvinium, and prochlorperazine - have not been reported for the treatment of MB; 286 however, several studies have reported their treatment efficacies in other cancers. 287 Prochlorperazine and trichostatin A were used for treating glioblastoma and pyrvinium 288 for the treatment of ovarian cancer (Horing et al. 2013; Otreba \& Buszman 2018; Zhang 289 et al. 2017) (33-35). Although the six small-molecule compounds

290

291 292 293 294 295 296 297 298 299 300 301 302 304

303 In summary, most single data set analyses are limited by the small sample size, high

obtained have not been adequately studied in MB. CMap is an online tool that provides $r$ esearchers with an index based on disease DEGs to search for potential therapeutic drugs. Recently, some traditional drugs have been found to have new therapeutic effects. The $d$ evelopment of new uses of old drugs can more quickly understand the pharmacological $p$ roperties of drugs, which will help their early application in clinical treatment. For examp le, atorvastatin is a well-known traditional lipid-

lowering drug, but in recent years, it has been found to have obvious therapeutic effects o $\mathrm{n}$ chronic subdural hematoma (Jiang et al. 2018). Metformin is a traditional an antidiabetic drug. In recent years, new therapeutic effects have been discovered in human dis eases such as anti-tumor effects, neuroprotective effects, etc (Podhorecka et al. 2017; Saewanee et al. 2019). So we have reason to speculate that the small-molecule drugs identified by CMap analysis may be potential drugs for the treatment of MB.

\section{Conclusions} experimental error, and lack of ethnic differences, and therefore cannot reliably identify 
305 the important genes and pathways involved in the pathogenesis of diseases. In this study,

306 the above problems were well avoided by using multiple data sets and integrated analysis

307 to improve the reliability and accuracy of the results. We identified 104 DEGs from four

308 groups of gene expression profiling data of MB and analyzed them comprehensively. The

309 hub genes identified by PPI network analysis include SYN1, CNTN2, FAIM2, MT3, and

$310 S H 3 G L 2$, which are involved in the pathogenesis of different cancers. We also identified

311 several small-molecule compounds that may have potential therapeutic effects on MB.

312 These findings provide new insights into the pathogenesis of MB and provide a basis for

313 treatment. however, further experimental verification is needed.

314 Acknowledgements

315 Not applicable.

316 References

317 Abdelfattah N, Rajamanickam S, Panneerdoss S, Timilsina S, Yadav P, Onyeagucha BC, Garcia M, Vadlamudi R, Chen Y, Brenner A, Houghton P, and Rao MK. 2018. MiR-584$5 p$ potentiates vincristine and radiation response by inducing spindle defects and DNA damage in medulloblastoma. Nat Commun 9:4541. 10.1038/s41467-018-06808-8

320

321

322

323

324

325

326

327

328

329

330

331

332

333

334

335

336

337

338

339

340

341

342

Bruntz RC, Taylor HE, Lindsley CW, and Brown HA. 2014. Phospholipase D2 mediates survival signaling through direct regulation of Akt in glioblastoma cells. J Biol Chem 289:600-616. 10.1074/jbc.M113.532978

Chen SM, Lin TK, Tseng YY, Tu CH, Lui TN, Huang SF, Hsieh LL, and Li YY. 2018. Targeting inhibitors of apoptosis proteins suppresses medulloblastoma cell proliferation via G2/M phase arrest and attenuated neddylation of p21. Cancer Med 7:3988-4003. 10.1002/cam4.1658

de Groot M, Aronica E, Heimans JJ, and Reijneveld JC. 2011. Synaptic vesicle protein 2A predicts response to levetiracetam in patients with glioma. Neurology 77:532-539. 10.1212/WNL.0b013e318228c110

Duan J, Ying Z, Su Y, Lin F, and Deng Y. 2017. alpha-Synuclein binds to cytoplasmic vesicles in U251 glioblastoma cells. Neurosci Lett 642:148-152. 10.1016/j.neulet.2017.01.067

Fang C, Huang Y, Pei Y, Zhang HH, Chen X, Guo H, Li S, Ji X, and Hu J. 2017. Genome-wide gene expression profiling reveals that CD274 is up-regulated new-onset type 1 diabetes mellitus. Acta Diabetol 54:757-767. 10.1007/s00592-017-1005-y

Franceschini A, Szklarczyk D, Frankild S, Kuhn M, Simonovic M, Roth A, Lin J, Minguez P, Bork $P$, von Mering C, and Jensen LJ. 2013. STRING v9.1: protein-protein interaction networks, with increased coverage and integration. Nucleic Acids Res 41:D808-815. 10.1093/nar/gks1094

Griesinger AM, Birks DK, Donson AM, Amani V, Hoffman LM, Waziri A, Wang M, Handler MH, and Foreman NK. 2013. Characterization of distinct immunophenotypes across pediatric brain tumor types. J Immunol 191:4880-4888. 10.4049/jimmunol.1301966 
343

344

345

346

347

348

349

350

351

352

353

354

355

356

357

358

359

360

361

362

363

364

365

366

367

368

369

370

371

372

373

374

375

376

377

378

379

380

381

382

383

384

385

386

387

388

389

390

391

392

393

Henriquez NV, Forshew T, Tatevossian R, Ellis M, Richard-Loendt A, Rogers H, Jacques TS, Reitboeck PG, Pearce K, Sheer D, Grundy RG, and Brandner S. 2013. Comparative expression analysis reveals lineage relationships between human and murine gliomas and a dominance of glial signatures during tumor propagation in vitro. Cancer Res 73:5834-5844. 10.1158/0008-5472.CAN-13-1299

Horing E, Podlech O, Silkenstedt B, Rota IA, Adamopoulou E, and Naumann U. 2013. The histone deacetylase inhibitor trichostatin a promotes apoptosis and antitumor immunity in glioblastoma cells. Anticancer Res 33:1351-1360.

Huang da W, Sherman BT, and Lempicki RA. 2009. Systematic and integrative analysis of large gene lists using DAVID bioinformatics resources. Nat Protoc 4:44-57. 10.1038/nprot.2008.211

Jiang R, Zhao S, Wang R, Feng H, Zhang J, Li X, Mao Y, Yuan X, Fei Z, Zhao Y, Yu X, Poon WS, Zhu X, Liu N, Kang D, Sun T, Jiao B, Liu X, Yu R, Zhang J, Gao G, Hao J, Su N, Yin G, Zhu X, Lu Y, Wei J, Hu J, Hu R, Li J, Wang D, Wei H, Tian Y, Lei P, Dong JF, and Zhang J. 2018. Safety and Efficacy of Atorvastatin for Chronic Subdural Hematoma in Chinese Patients: A Randomized ClinicalTrial. JAMA Neurol 75:1338-1346. 10.1001/jamaneurol.2018.2030

Jin Y, Xiong A, Zhang Z, Li S, Huang H, Yu TT, Cao X, and Cheng SY. 2014. MicroRNA-31 suppresses medulloblastoma cell growth by inhibiting DNA replication through minichromosome maintenance 2. Oncotarget 5:4821-4833. 10.18632/oncotarget.2043

Kang HC, Kim JI, Chang HK, Woodard G, Choi YS, Ku JL, Jablons DM, and Kim IJ. 2016. FAIM2, as a novel diagnostic maker and a potential therapeutic target for small-cell lung cancer and atypical carcinoid. Sci Rep 6:34022. 10.1038/srep34022

Kim SY, Sung KW, Hah JO, Yoo KH, Koo HH, Kang HJ, Park KD, Shin HY, Ahn HS, Im HJ, Seo JJ, Lim YJ, Lee YH, Shin HJ, Lim DH, Cho BK, Ra YS, and Choi JU. 2010. Reduceddose craniospinal radiotherapy followed by high-dose chemotherapy and autologous stem cell rescue for children with newly diagnosed high-risk medulloblastoma or supratentorial primitive neuroectodermal tumor. Korean J Hematol 45:120-126. 10.5045/kjh.2010.45.2.120

Lamb J, Crawford ED, Peck D, Modell JW, Blat IC, Wrobel MJ, Lerner J, Brunet JP, Subramanian A, Ross KN, Reich M, Hieronymus H, Wei G, Armstrong SA, Haggarty SJ, Clemons PA, Wei R, Carr SA, Lander ES, and Golub TR. 2006. The Connectivity Map: using gene-expression signatures to connect small molecules, genes, and disease. Science 313:1929-1935. 10.1126/science.1132939

Liu H, Sun Q, Zhang M, Zhang Z, Fan X, Yuan H, Li C, Guo Y, Ning W, Sun Y, Song Y, and Yu C. 2017. Differential expression of folate receptor 1 in medulloblastoma and the correlation with clinicopathological characters and target therapeutic potential. Oncotarget 8:23048-23060. 10.18632/oncotarget.15480

Luis Albasanz J, Fernandez M, and Martin M. 2002. Internalization of metabotropic glutamate receptor in C6 cells through clathrin-coated vesicles. Brain Res Mol Brain Res 99:54-66. 10.1016/s0169-328x(02)00103-1

Muscal JA, Scorsone KA, Zhang L, Ecsedy JA, and Berg SL. 2013. Additive effects of vorinostat and MLN8237 in pediatric leukemia, medulloblastoma, and neuroblastoma cell lines. Invest New Drugs 31:39-45. 10.1007/s10637-012-9831-9

Oehler C, von Bueren AO, Furmanova P, Broggini-Tenzer A, Orlowski K, Rutkowski S, Frei K, Grotzer MA, and Pruschy M. 2011. The microtubule stabilizer patupilone (epothilone B) is a potent radiosensitizer in medulloblastoma cells. Neuro Oncol 13:1000-1010. $10.1093 /$ neuonc/nor069

Otreba M, and Buszman E. 2018. Perphenazine and prochlorperazine induce concentrationdependent loss in human glioblastoma cells viability. Pharmazie 73:19-21. 10.1691/ph.2018.7806

Peer) reviewing PDF | (2019:10:41888:1:1:NEW 15 Jan 2020) 
394

395

396

397

398

399

400

401

402

403

404

405

406

407

408

409

410

411

412

413

414

415

416

417

418

419

420

421

422

423

424

425

426

427

428

429

430

431

432

433

434

435

436

437

438

439

440

441

442

443

Podhorecka M, Ibanez B, and Dmoszynska A. 2017. Metformin - its potential anti-cancer and anti-aging effects. Postepy Hig Med Dosw (Online) 71:170-175. 10.5604/01.3001.0010.3801

Rivero-Hinojosa S, Lau LS, Stampar M, Staal J, Zhang H, Gordish-Dressman H, Northcott PA, Pfister SM, Taylor MD, Brown KJ, and Rood BR. 2018. Proteomic analysis of Medulloblastoma reveals functional biology with translational potential. Acta Neuropathol Commun 6:48. 10.1186/s40478-018-0548-7

Saewanee N, Praputpittaya T, Malaiwong N, Chalorak P, and Meemon K. 2019. Neuroprotective effect of metformin on dopaminergic neurodegeneration and alphasynuclein aggregation in C. elegans model of Parkinson's disease. Neurosci Res. 10.1016/j.neures.2019.12.017

Shaabanpour Aghamaleki F, Mollashahi B, Aghamohammadi N, Rostami N, Mazloumi Z, Mirzaei H, Moradi A, Sheikhpour M, and Movafagh A. 2019. Bioinformatics Analysis of Key Genes and Pathways for Medulloblastoma as a Therapeutic Target. Asian Pac J Cancer Prev 20:221-227. 10.31557/APJCP.2019.20.1.221

Tao YF, Xu LX, Lu J, Cao L, Li ZH, Hu SY, Wang NN, Du XJ, Sun LC, Zhao WL, Xiao PF, Fang F, Li YH, Li G, Zhao H, Li YP, Xu YY, Ni J, Wang J, Feng X, and Pan J. 2014. Metallothionein III (MT3) is a putative tumor suppressor gene that is frequently inactivated in pediatric acute myeloid leukemia by promoter hypermethylation. $J$ Transl Med 12:182. 10.1186/1479-5876-12-182

Tuncel G, and Kalkan R. 2018. Receptor tyrosine kinase-Ras-PI 3 kinase-Akt signaling network in glioblastoma multiforme. Med Oncol 35:122. 10.1007/s12032-018-1185-5

Vogelstein B, Papadopoulos N, Velculescu VE, Zhou S, Diaz LA, Jr., and Kinzler KW. 2013. Cancer genome landscapes. Science 339:1546-1558. 10.1126/science.1235122

Wang J, Garancher A, Ramaswamy V, and Wechsler-Reya RJ. 2018. Medulloblastoma: From Molecular Subgroups to Molecular Targeted Therapies. Annu Rev Neurosci 41:207-232. 10.1146/annurev-neuro-070815-013838

Wang Q, Li H, Liu N, Chen XY, Wu ML, Zhang KL, Kong QY, and Liu J. 2008. Correlative analyses of notch signaling with resveratrol-induced differentiation and apoptosis of human medulloblastoma cells. Neurosci Lett 438:168-173. 10.1016/j.neulet.2008.04.012

Wortham M, Guo C, Zhang M, Song L, Lee BK, lyer VR, Furey TS, Crawford GE, Yan H, and He Y. 2014. Chromatin accessibility mapping identifies mediators of basal transcription and retinoid-induced repression of OTX2 in medulloblastoma. PLoS One 9:e107156. 10.1371/journal.pone.0107156

Xi Y, Tang W, Yang S, Li M, He Y, and Fu X. 2017. Mining the glioma susceptibility genes in children from gene expression profiles and a methylation database. Oncol Lett 14:34733479. 10.3892/ol.2017.6579

Yaghi L, Poras I, Simoes RT, Donadi EA, Tost J, Daunay A, de Almeida BS, Carosella ED, and Moreau P. 2016. Hypoxia inducible factor-1 mediates the expression of the immune checkpoint HLA-G in glioma cells through hypoxia response element located in exon 2. Oncotarget 7:63690-63707. 10.18632/oncotarget.11628

Yan Y, and Jiang Y. 2016. RACK1 affects glioma cell growth and differentiation through the CNTN2-mediated RTK/Ras/MAPK pathway. Int J Mol Med 37:251-257. 10.3892/ijmm.2015.2421

Yucebas M, Yilmaz Susluer S, Onur Caglar H, Balci T, Dogan Sigva ZO, Akalin T, Oktar N, Dalbasti T, Biray Avci C, and Gunduz C. 2016. Expression profiling of RE1-silencing transcription factor (REST), REST corepressor 1 (RCOR1), and Synapsin 1 (SYN1) genes in human gliomas. J BUON 21:964-972.

Zeng WJ, Yang YL, Liu ZZ, Wen ZP, Chen YH, Hu XL, Cheng Q, Xiao J, Zhao J, and Chen XP. 2018. Integrative Analysis of DNA Methylation and Gene Expression Identify a Three-

Peer] reviewing PDF | (2019:10:41888:1:1:NEW 15 Jan 2020) 
444 Gene Signature for Predicting Prognosis in Lower-Grade Gliomas. Cell Physiol Biochem $445 \quad$ 47:428-439. 10.1159/000489954

446 Zhang C, Zhang Z, Zhang S, Wang W, and Hu P. 2017. Targeting of Wnt/beta-Catenin by

447

448

449

450

451 Anthelmintic Drug Pyrvinium Enhances Sensitivity of Ovarian Cancer Cells to Chemotherapy. Med Sci Monit 23:266-275. 10.12659/msm.901667

Zhu Y, Zhang X, Wang L, Ji Z, Xie M, Zhou X, Liu Z, Shi H, and Yu R. 2017. Loss of SH3GL2 promotes the migration and invasion behaviours of glioblastoma cells through activating the STAT3/MMP2 signalling. J Cell Mol Med 21:2685-2694. 10.1111/jcmm.13184

452 


\section{Figure 1}

Using the Venny map to obtain common DEGs in MB, and the cross areas represented the commonly DEGs of the four datasets (GSE42656, GSE109401, GSE50161, and GSE74195).

A) Commonly up-regulated genes(29 DEGs); B) Commonly down-regulated genes(75 DEGs).

A

GSE42656

GSE74195

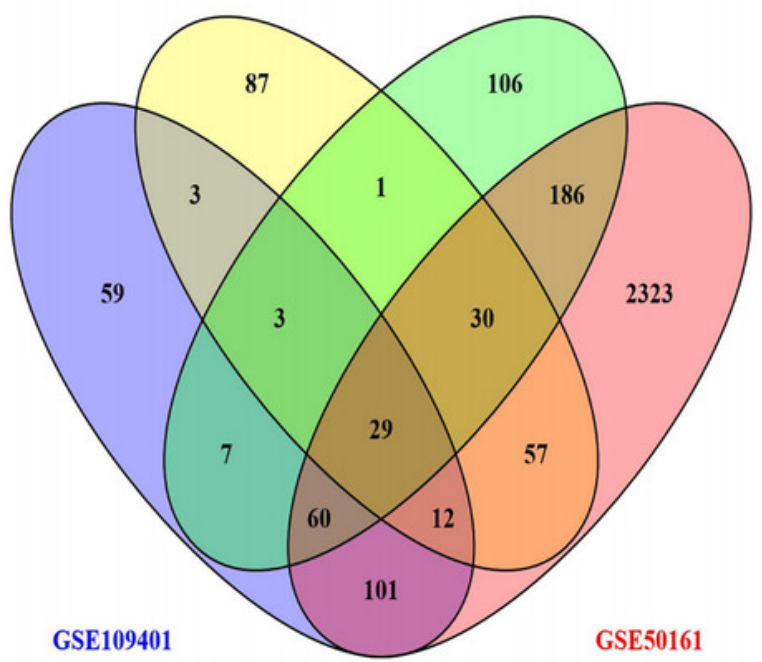

B

GSE42656

GSE74195

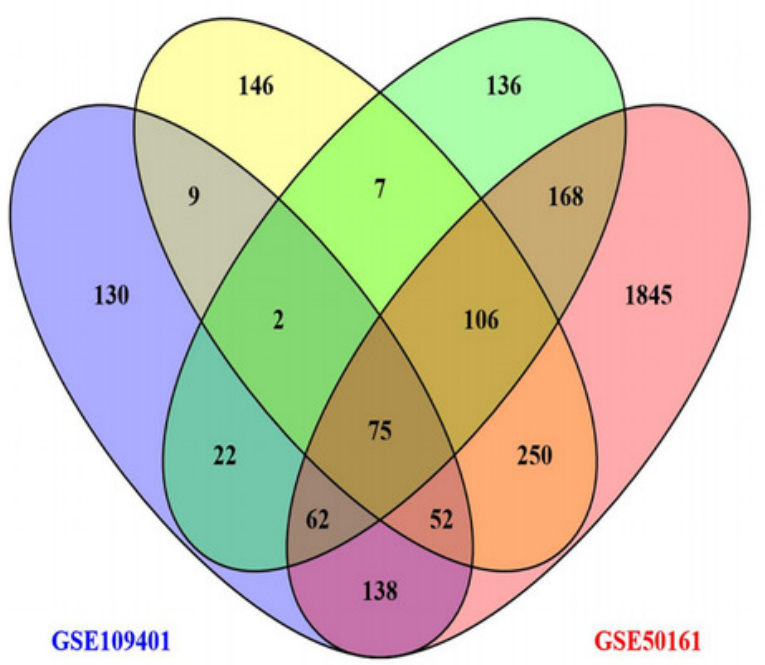


Figure 2

Gene Ontology (GO) functional annotation analysis and significant terms of DEGs in MB and $\mathrm{GO}$ analysis divided into three parts.

cyan for cell component, pink for molecular function, and blue for biological process

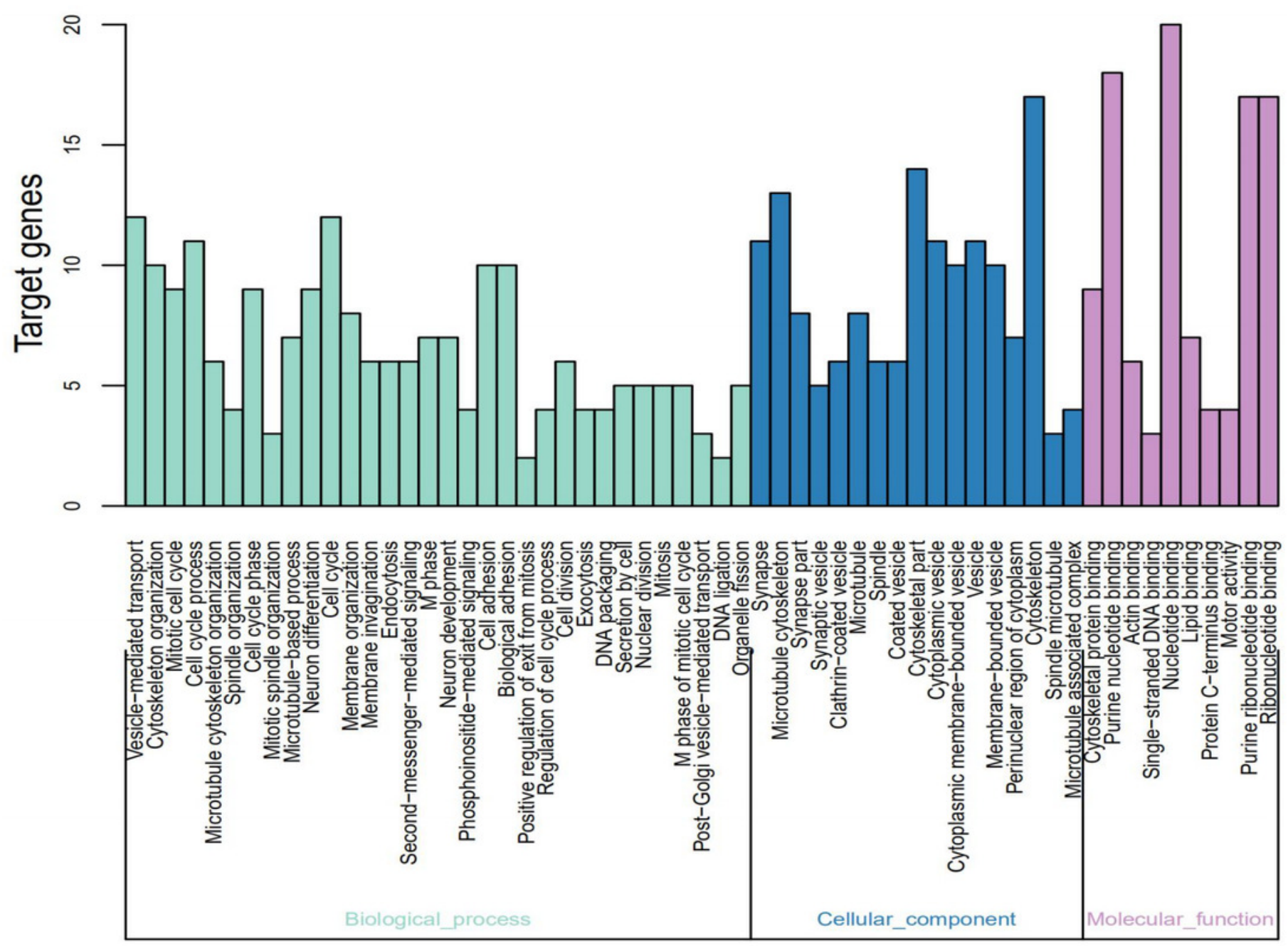


Figure 3

Kyoto Encyclopedia of Genes and Genomes (KEGG) signal pathway analysis of DEGs in MB.

Red represent the common up-regulated genes and green represent the common downregulated genes

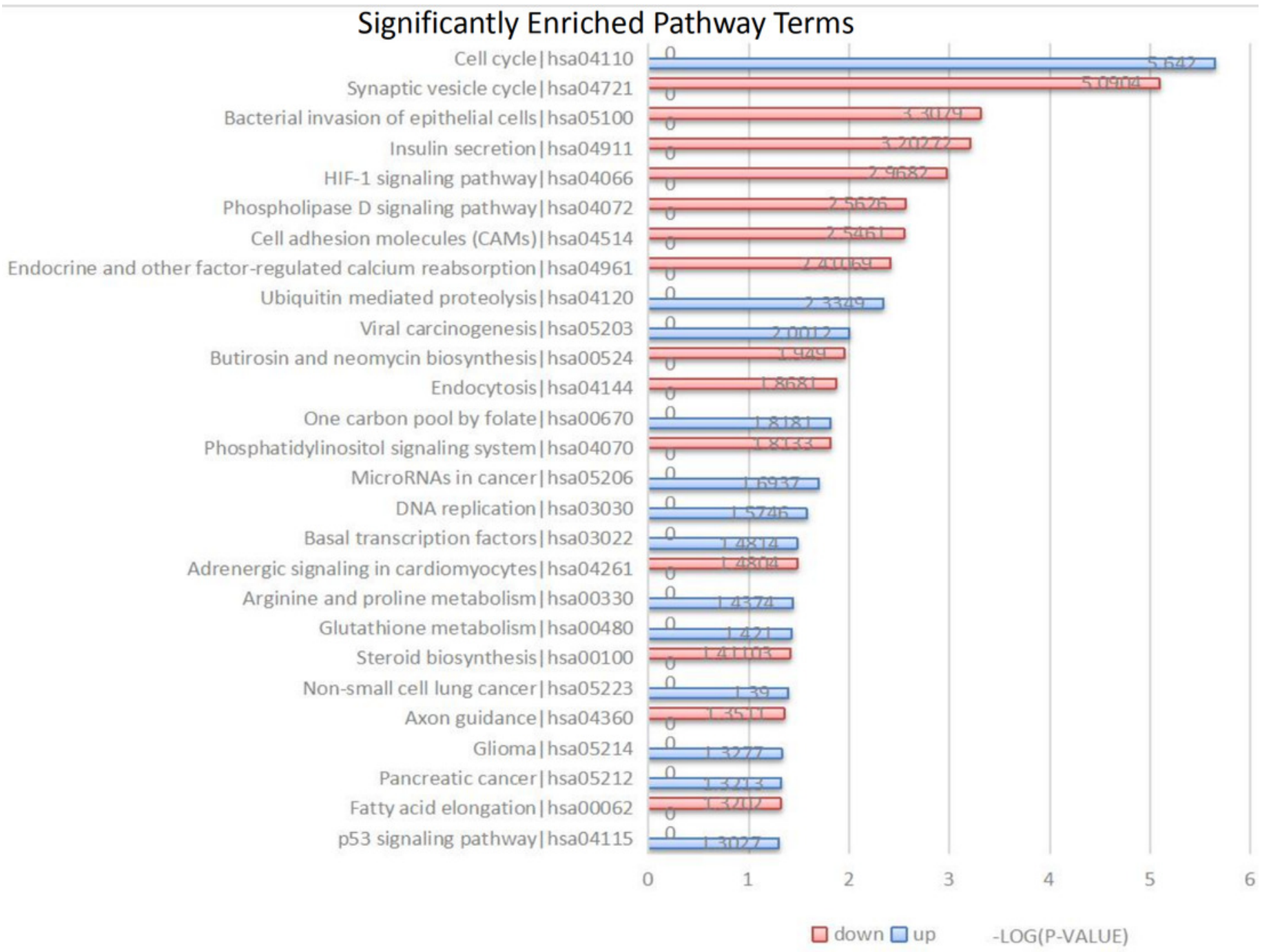


Figure 4

The PPI network of DEGs.

(A) 100 DEGs were incorporated into the network consisted of 29 upregulated (red) and 71 downregulated (green) genes; (B) Hub genes determined by the degree of connectivity between DEGs.

A

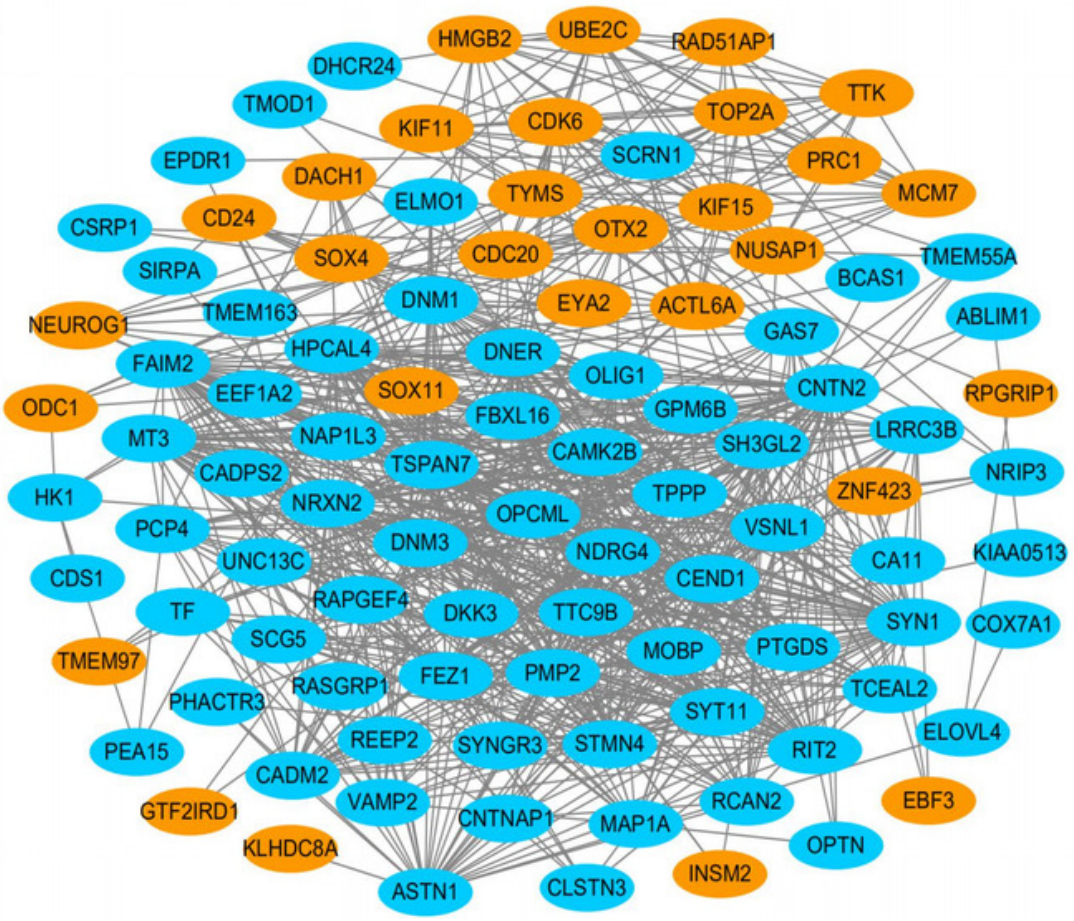

B

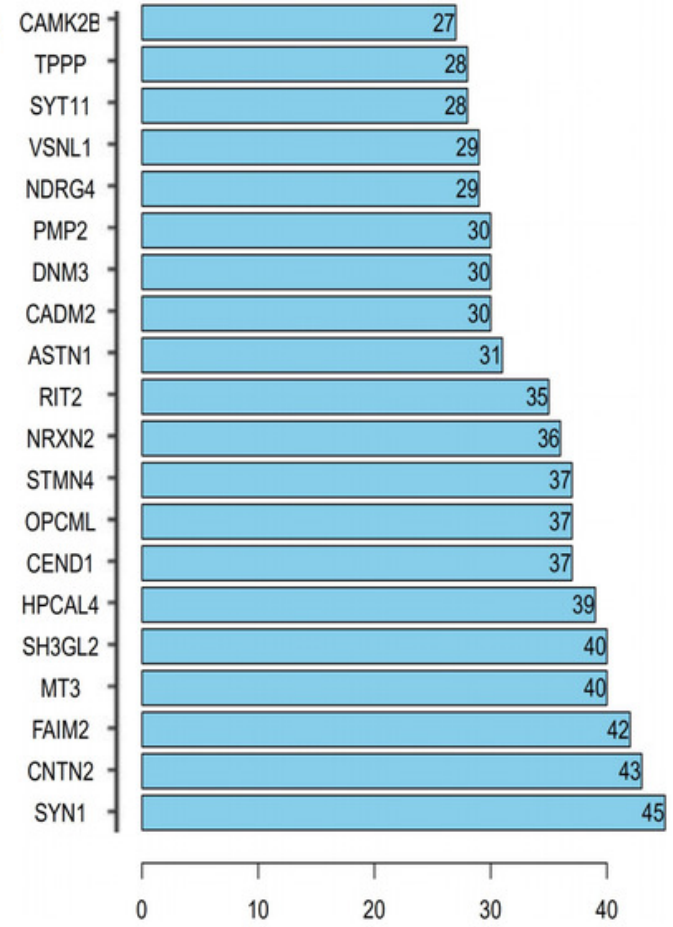


Figure 5

The 3D structures of five small-molecule compounds identified as potential drugs for MB treatment.

(A) Vorinostat; (B) Resveratrol; (C) Trichostatin A; (D) Pyrvinium, and (E) Prochlorperazine.

A

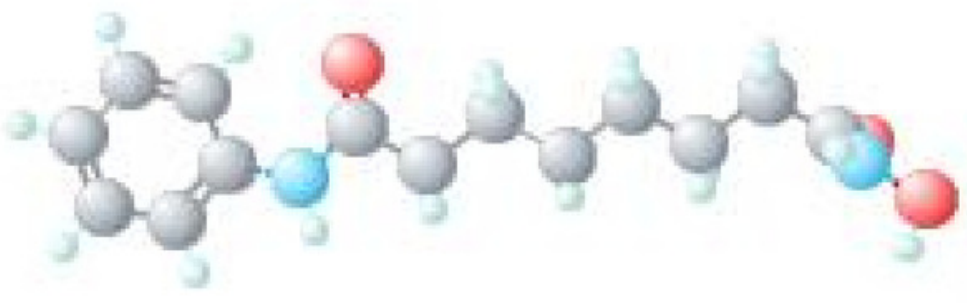

B

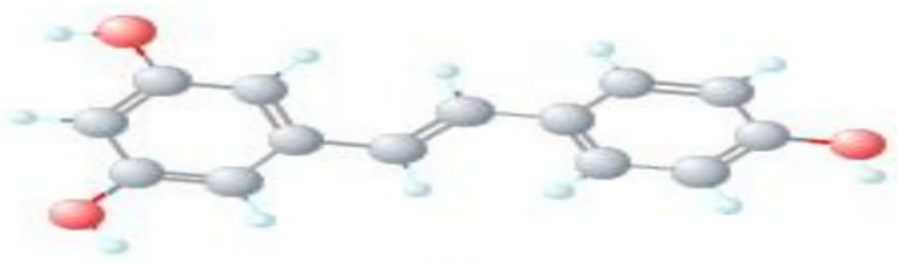

C

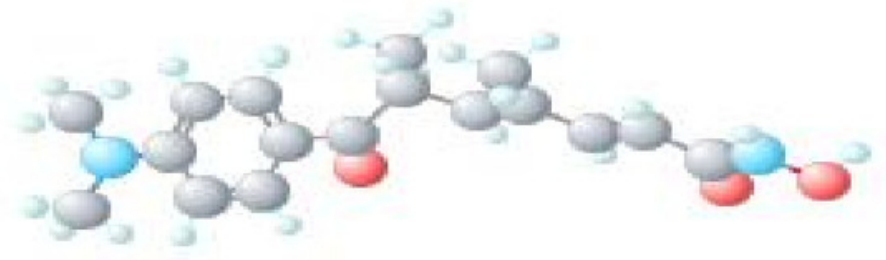

D

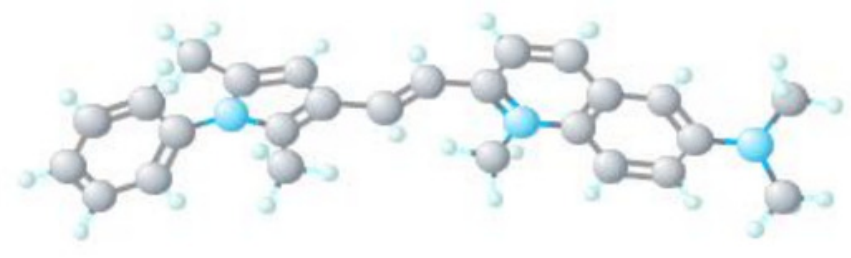

$\mathbf{E}$

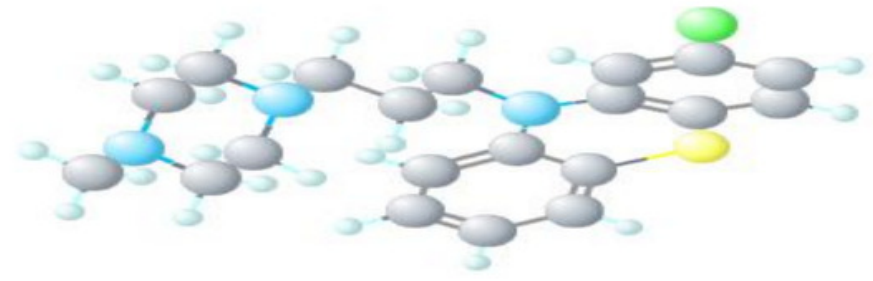

Vorinostat

MolecularFormula: $\mathrm{C}_{14} \mathrm{H}_{20} \mathrm{~N}_{2} \mathrm{O}_{3}$

PubChem CID: 5311

Resveratrol

Molecular Formula: $\mathrm{C}_{14} \mathrm{H}_{12} \mathrm{O}_{3}$

PubChem CID: 445154

Trichostatin A

Molecular Formula: $\mathrm{C}_{17} \mathrm{H}_{22} \mathrm{~N}_{2} \mathrm{O}_{3}$

PubChem CID: 444732

Pyrvinum

Molecular Formula: $\mathrm{C}_{26} \mathrm{H}_{28} \mathrm{~N}_{3}{ }^{+}$

PubChem CID:5281035

Prochlorperazine

Molecular Formula: $\mathrm{C}_{20} \mathrm{H}_{24} \mathrm{C}_{1} \mathrm{~N}_{3} \mathrm{~S}$ PubChem CID:4917 
Figure 6

TheexpressionlevelsofthefivehubgenesinthePPInetworkweredetectedby RT-qPCR.

TheseincludedSYN1,CNTN2,FAIM2,MT3, andSH3GL2(P<0.05).

A

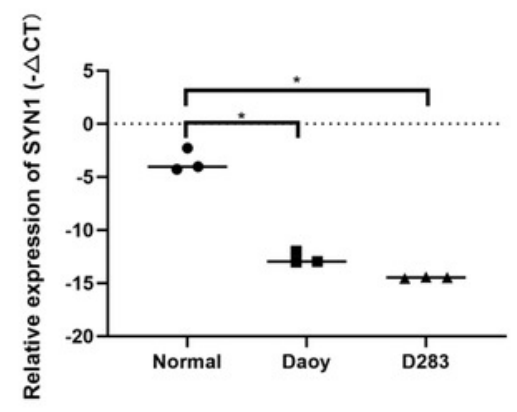

$\mathrm{D}$

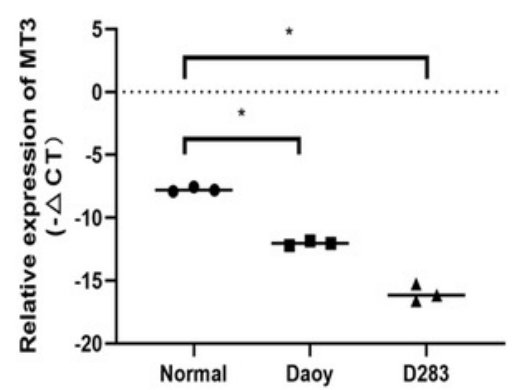

B

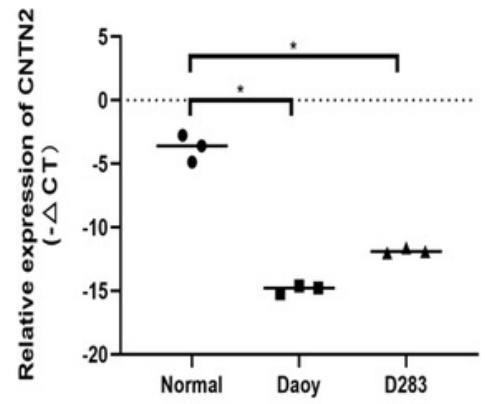

$\mathrm{E}$

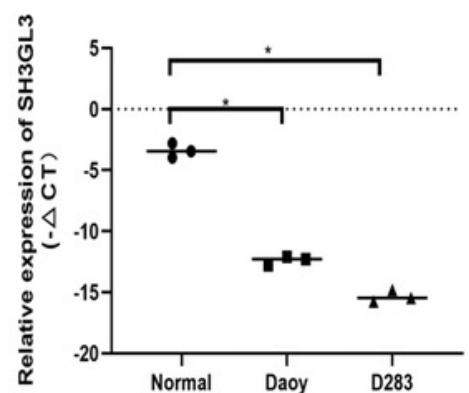

C

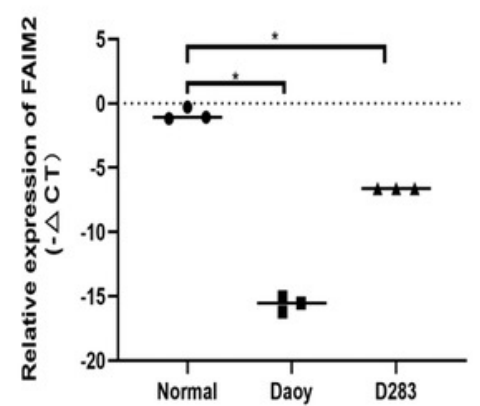




\section{Table $\mathbf{1}$ (on next page)}

The 104 differentially expressed genes (DEGs), including 29 upregulated genes and 75 downregulated genes were identified in the medulloblastoma tissues from four profile datasets using normal brain tissues as a reference.

Abbreviation:DEGs, differentially expressed genes. 
1 Table I .The 104 differentially expressed genes (DEGs), including 29 upregulated genes and 75 2 downregulated genes were identified in the medulloblastoma tissues from four profile datasets 3 using normal brain tissues as a reference.

\begin{tabular}{ll}
\hline DEGs & \multicolumn{1}{c}{ Gene Name } \\
\hline Up-regulated 29 & ACTL6A,UBE2C,TMEM97,GTF2IRD1,INSM2,CDC20,PRC1,ZNF423,HMGB2,ODC1,MCM7,KL \\
& HDC8A,SOX11,RAD51AP1,KIF15,CDK6,EBF3,EYA2,TYMS,TTK,CD24,DACH1,SOX4,NUSAP \\
& 1,RPGRIP1,KIF11,NEUROG1,OTX2,TOP2A \\
\hline & CADPS2,TMEM163,UNC13C,DNER,CNTNAP1,DNM3,TMOD1,FEZ1,RAPGEF4,RIT2,NDRG4, \\
& TPPP,TMEM55A,MT3,FAIM2,ABLIM1,RCAN2,MAP1A,DHCR24,NRXN2,PTGDS,CDS1,RASG \\
& RP1,PCP4,NRIP3,HPCAL4,PTCHD1,GAS7,KIAA0513,PMP2,PHACTR3,TF,CADM2,CNTN2,VS \\
& NL1,BCAS1,DKK3,SCRN1,FBXL16,ELOVL4,OPCML,DNM1,EEF1A2,TTC9B,CA11,CEND1,M \\
& OBP,SH3GL2,SCG5,EPDR1,LRRC3B,COX7A1,NAP1L3,TSPAN7,CAMK2B,OPTN,STMN4,CLS \\
Down-regulated 75 & TN3,PEA15,SYN1,RNF175,REEP2,CSRP1,SYT11,TCEAL2,GPM6B,TPRG1L,ASTN1,MAP7D2,S \\
& IRPA,OLIG1,SYNGR3,ELMO1,HK1,VAMP2 \\
\hline
\end{tabular}

Abbreviation:DEGs, differentially expressed genes. 


\section{Table 2 (on next page)}

DEGs divided into upregulated genes and downregulated genes and their first ten meaningful GO enrichment analyses.

Abbreviation: GO, Gene Ontology; DEGs, differentially expressed genes. 
1 Table II. DEGs divided into upregulated genes and downregulated genes and their first ten

2 meaningful GO enrichment analyses.

\begin{tabular}{|c|c|c|c|}
\hline Term & Description & Count & P-Value \\
\hline \multicolumn{4}{|l|}{ Up-regulated } \\
\hline GO:0005819 & Spindle & 6 & $1.92 \mathrm{E}-06$ \\
\hline GO:0000278 & Mitotic cell cycle & 8 & $5.99 \mathrm{E}-06$ \\
\hline GO:0003677 & DNA binding & 15 & $1.12 \mathrm{E}-05$ \\
\hline GO:0022403 & Cell cycle phase & 8 & $1.25 \mathrm{E}-05$ \\
\hline GO:0000279 & M phase & 7 & $3.80 \mathrm{E}-05$ \\
\hline GO:0022402 & Cell cycle process & 8 & $9.14 \mathrm{E}-05$ \\
\hline GO:0007049 & Cell cycle & 9 & $9.45 \mathrm{E}-05$ \\
\hline GO:0007051 & Spindle organization & 4 & $9.51 \mathrm{E}-05$ \\
\hline GO:0015630 & Microtubule cytoskeleton & 7 & $1.03 \mathrm{E}-04$ \\
\hline GO:0007017 & Microtubule-based process & 6 & $1.27 \mathrm{E}-04$ \\
\hline \multicolumn{4}{|c|}{ Down-regulated } \\
\hline GO:0045202 & Synapse & 11 & $5.27 \mathrm{E}-06$ \\
\hline GO:0016192 & Vesicle-mediated transport & 11 & $5.69 \mathrm{E}-05$ \\
\hline GO:0044456 & Synapse part & 8 & $1.45 \mathrm{E}-04$ \\
\hline GO:0030136 & Clathrin-coated vesicle & 6 & $3.77 \mathrm{E}-04$ \\
\hline GO:0008021 & Synaptic vesicle & 5 & 4.37E-04 \\
\hline GO:0031410 & Cytoplasmic vesicle & 11 & 7.37E-04 \\
\hline GO:0030135 & Coated vesicle & 6 & $8.80 \mathrm{E}-04$ \\
\hline GO:0016023 & Cytoplasmic membrane-bounded vesicle & 10 & $9.86 \mathrm{E}-04$ \\
\hline GO:0031982 & Vesicle & 11 & 0.001022664 \\
\hline GO:0031988 & Membrane-bounded vesicle & 10 & 0.001235649 \\
\hline
\end{tabular}




\section{Table 3(on next page)}

KEGG pathway analysis of upregulated and downregulated genes in MB

Abbreviation: KEGG, Kyoto Encyclopedia of Genes and Genomes; ID,identification. 
1 Table III. KEGG pathway analysis of upregulated and downregulated genes in MB

\begin{tabular}{|c|c|c|c|c|c|}
\hline Database & ID & Term & Gene Count & P-Value & Gene Names \\
\hline \multicolumn{6}{|l|}{ Up-regulated } \\
\hline KEGG PATHWAY & hsa04110 & Cell cycle & 4 & $2.28 \mathrm{E}-06$ & $\begin{array}{l}\text { TTK、CDC20、MCM7、 } \\
\text { CDK6 }\end{array}$ \\
\hline KEGG PATHWAY & hsa04120 & Ubiquitin mediated proteolysis & 2 & 0.004624353 & CDC20、UBE2C \\
\hline KEGG PATHWAY & hsa05203 & Viral carcinogenesis & 2 & 0.009970546 & CDC20、CDK6 \\
\hline KEGG PATHWAY & hsa00670 & One carbon pool by folate & 1 & 0.015198488 & TYMS \\
\hline KEGG PATHWAY & hsa05206 & MicroRNAs in cancer & 2 & 0.020243092 & CDK6、SOX4 \\
\hline KEGG PATHWAY & hsa03030 & DNA replication & 1 & 0.026628344 & MCM7 \\
\hline KEGG PATHWAY & hsa03022 & Basal transcription factors & 1 & 0.033001239 & GTF2IRD1 \\
\hline KEGG PATHWAY & hsa00330 & Arginine and proline metabolism & 1 & 0.03652431 & ODC1 \\
\hline KEGG PATHWAY & hsa00480 & Glutathione metabolism & 1 & 0.037930065 & ODC1 \\
\hline KEGG PATHWAY & hsa05223 & Non-small cell lung cancer & 1 & 0.040735637 & CDK6 \\
\hline \multicolumn{6}{|l|}{ Down-regulated } \\
\hline KEGG PATHWAY & hsa04721 & Synaptic vesicle cycle & 4 & $8.12 \mathrm{E}-06$ & $\begin{array}{l}\text { DNM3、UNC13C、DNM1、 } \\
\text { VAMP2 }\end{array}$ \\
\hline KEGG PATHWAY & hsa05100 & $\begin{array}{l}\text { Bacterial invasion of epithelial } \\
\text { cells }\end{array}$ & 3 & 0.000492126 & DNM3、DNM1、ELMO1 \\
\hline KEGG PATHWAY & hsa04911 & Insulin secretion & 3 & 0.000627009 & $\begin{array}{l}\text { RAPGEF4、CAMK2B、 } \\
\text { VAMP2 }\end{array}$ \\
\hline KEGG PATHWAY & hsa04066 & HIF-1 signaling pathway & 3 & 0.001075892 & HK1、TF、CAMK2B \\
\hline KEGG PATHWAY & hsa04072 & $\begin{array}{l}\text { Phospholipase D signaling } \\
\text { pathway }\end{array}$ & 3 & 0.002737401 & DNM3、RAPGEF4、DNM1 \\
\hline KEGG PATHWAY & hsa04514 & Cell adhesion molecules (CAMs) & 3 & 0.002843803 & $\begin{array}{l}\text { NRXN2、CNTN2、 } \\
\text { CNTNAP1 }\end{array}$ \\
\hline KEGG PATHWAY & hsa04961 & $\begin{array}{l}\text { Endocrine and other Factor- } \\
\text { regulated calcium reabsorption }\end{array}$ & 2 & 0.003884233 & DNM3、DNM1 \\
\hline KEGG PATHWAY & hsa00524 & $\begin{array}{l}\text { Butirosin and neomycin } \\
\text { biosynthesis }\end{array}$ & 1 & 0.011244262 & HK1 \\
\hline KEGG PATHWAY & hsa04144 & Endocytosis & 3 & 0.01354801 & DNM3、DNM1、SH3GL2 \\
\hline KEGG PATHWAY & hsa04070 & $\begin{array}{l}\text { Phosphatidylinositol signaling } \\
\text { system }\end{array}$ & 2 & 0.0153706 & CDS1、TMEM55A \\
\hline
\end{tabular}

3 Abbreviation: KEGG, Kyoto Encyclopedia of Genes and Genomes; ID, identification.

4.

5 


\section{Table 4 (on next page)}

Six small-molecule compounds identified as potential drugs for MB treatment by CMap analysis

Abbreviation: CMap, connectivity map. 
1 Table IV. Six small-molecule compounds identified as potential drugs for MB treatment by

2 CMap analysis

\begin{tabular}{lll}
\hline Term & Enrichment & P-Value \\
\hline Vorinostat & -0.842 & 0 \\
Resveratrol & -0.827 & 0 \\
Trichostatin A & -0.568 & 0 \\
Pyrvinium & -0.727 & 0.00077 \\
Prochlorperazine & -0.475 & 0.00082 \\
$0175029-0000$ & -0.718 & 0.00099 \\
\hline
\end{tabular}

3 Abbreviation: CMap, connectivity map.

4 